\title{
Visualisation of Wear Debris from Orthopaedic Implants
}

\author{
A. BRZANA*
}

Minton Park, London Road, Amesbury, SP4 7RT, UK

NanoSight develops, manufactures and supplies a unique family of instruments for the visualisation and sizing of nanoparticles. Based on laser light scattering microscopy, our nanoparticle tracking analysis technology is an exciting new capability that offers significant advantages over existing light scattering techniques for the characterisation of polydispersed populations of nanoscale particles. Independent of particle density or refractive index, nanoparticle tracking analysis dynamically tracks individual particles within the range of 10-1000 nm, validates size distributions with a real-time view of particles, and provides a qualitative and quantitative analysis of sample concentration.

\section{Instruments}

The LM10 (Fig. 1a) and LM20 (Fig. 1b) systems enable users to view the sample under analysis and use our nanoparticle tracking analysis (NTA) technology to calculate the particle size and size distribution [1].

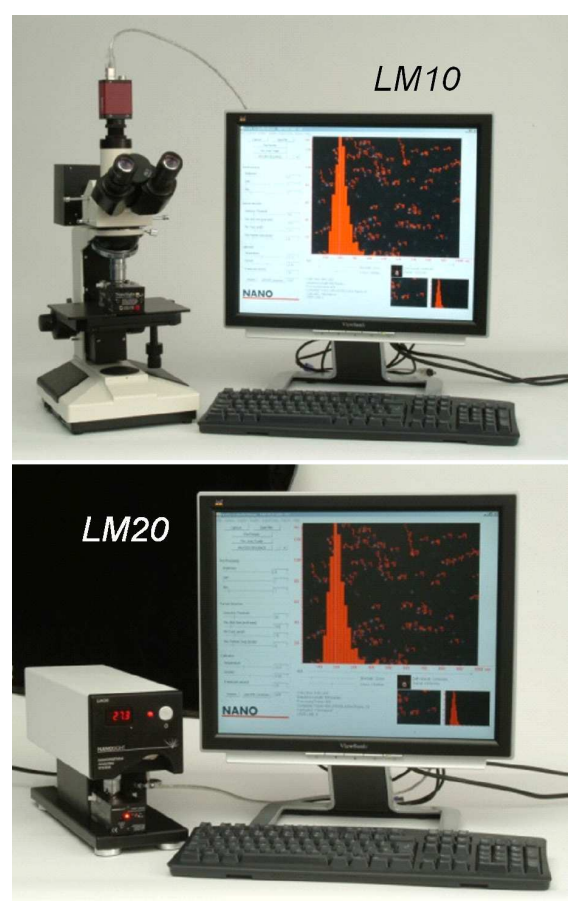

Fig. 1. NanoSight LM10 and NanoSight LM20.

* e-mail: agnieszka.brzana@nanosight.com; www.nanosight.com

\section{Technology}

The instrument uses a laser light source to illuminate nanoscale particles (Fig. 2). Enhanced by a near-perfect black background, particles appear individually as point-scatterers moving under Brownian motion. Polydisperse and multimodal systems are instantly recognisable and quantifiable, as are agglomerates and contaminants.

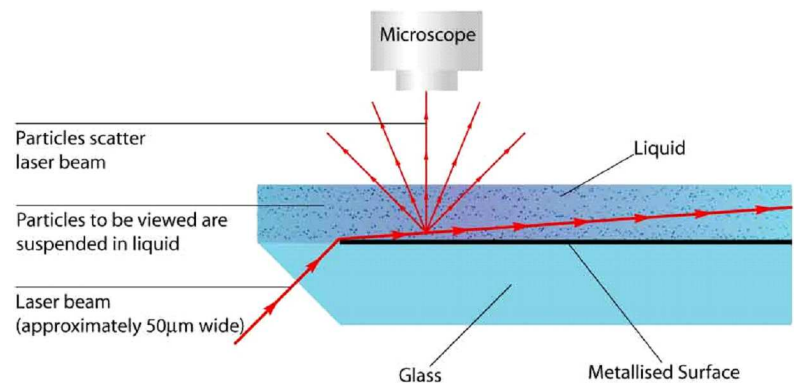

Fig. 2. Schematic showing the optical path of the laser beam and the detection objective viewing the beam through the window.

The image analysis NTA software automatically tracks and sizes the nanoparticles on a particle-by-particle basis. Results are displayed as a frequency size distribution and output to spreadsheet.

Video clips of images are recorded and retained for further analysis. This is an absolute method, so no calibration or refractive index data is required.

\section{Size determination and visualisation of wear debris from orthopaedic implants}

Characterising the size and quantity of wear debris produced from an orthopaedic implant is of importance when determining the biocompatibility and longevity of the implant.

The production and size of wear debris can influence factors such as:

- Hypersensitivity,

- Aseptic loosening of the implant and associated osteolysis,

- Systemic distribution and accumulation of implant debris,

- 3rd party degradation of articulating surfaces,

- Bioavailability and bioreactivity of metallic species,

- Design and choice of material for a specific implant. 


\section{Sample preparation}

Samples are prepared to remove the protein content from the synthetic/natural synovial fluid. Failure to remove the proteinatious content would increase the signal to noise ratio but would not prevent analysis. Polyethylene samples are treated for 1-2 days in $\mathrm{KOH}$ followed by solvent extraction using a chloroform/methanol mix. Hot enzymatic digestion is used to isolate metallic particles. In general, the technique requires sample dilution to approximately $10^{9}$ particles $/ \mathrm{ml}$. From this, a sample of less than $500 \mu \mathrm{l}$ is taken and injected into the viewing unit. The results are shown in the pictures below (Figs. 3-6) [2].

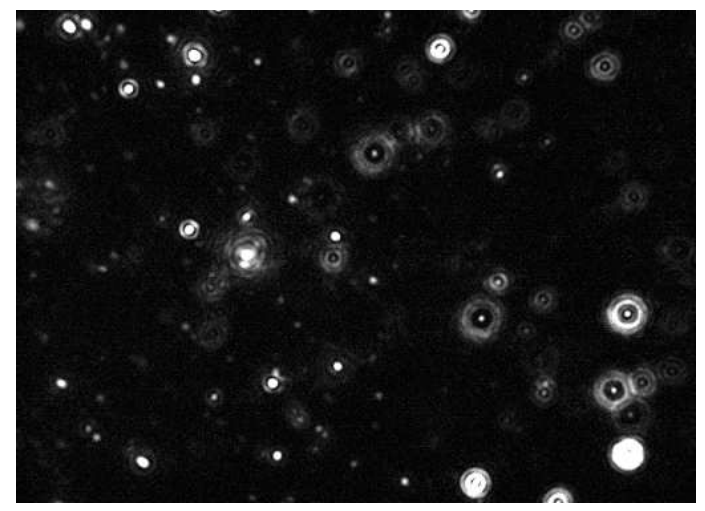

Fig. 3. Image of $\mathrm{CrN}$ wear debris as produced by the NanoSight system. The polydispersed nature of the sample can be clearly seen with some particles scattering more intensely.

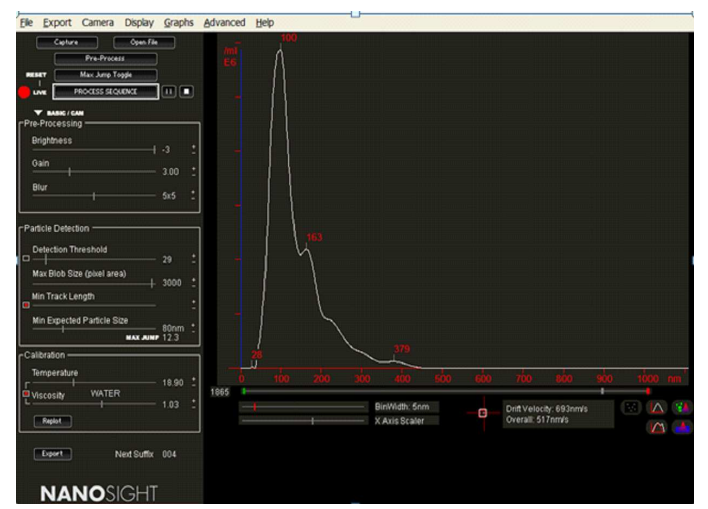

Fig. 4. Particle size distribution produced from a metal on metal $\mathrm{CrN}$ prosthesis.

\section{Summary}

NanoSight can provide particle size and concentration of wear debris in liquid suspension, following separation from the synovial fluid.

- Particles can be measured in their natural state (without drying/vacuum conditions).

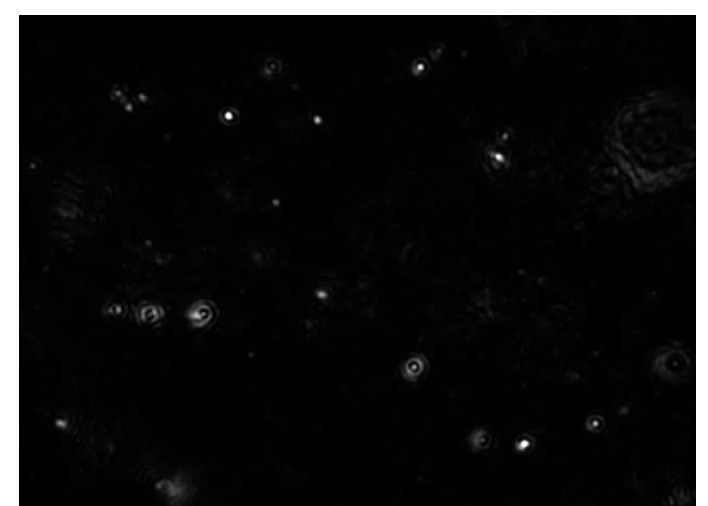

Fig. 5. Image of PE wear debris as produced by the NanoSight system.

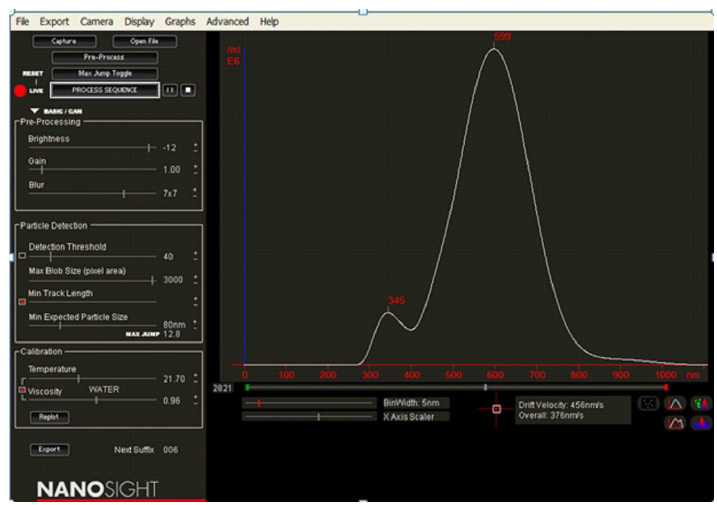

Fig. 6. Particle size distribution produced from a metal on metal PE prosthesis.

- Greater ability to size polydisperse samples due to the insensitivity to intensity (commonly associated with other light scattering techniques).

- Small sample volume.

- Low cost of unit.

- Visualisation of individual particles without any pre-treatment such as labelling.

- Straightforward technique and rapid results.

- Allows the study of time-based changes, such as agglomeration/stability.

- Accurate particle sizing in the range $10-1000 \mathrm{~nm}$.

\section{References}

[1] LM10 and LM20 www. nanosight.com .

[2] Nanosight application note: "Size Determination, Visualisation of Wear Debris from Orthopaedic Implants". 